\title{
Shell-model calculations for p-shell hypernuclei
}

\author{
D.J. Millener \\ Brookhaven National Laboratory, Upton, NY 11973, USA
}

\begin{abstract}
The interpretation of hypernuclear $\gamma$-ray data for p-shell hypernuclei in terms of shell-model calculations that include the coupling of $\Lambda$ - and $\Sigma$-hypernuclear states is briefly reviewed. Next, ${ }_{\Lambda}^{8} \mathrm{Li} /{ }_{\Lambda}^{8} \mathrm{Be}$ and ${ }_{\Lambda}^{9} \mathrm{Li}$ are considered, both to exhibit features of $\Lambda-\Sigma$ coupling and as possible source of observed, but unassigned, hypernuclear $\gamma$ rays. Then, the feasibility of measuring the ground-state doublet spacing of ${ }_{\Lambda}^{10} \mathrm{Be}$, which, like ${ }_{\Lambda}^{9} \mathrm{Li}$, could be studied via the $\left(K^{-}, \pi^{0} \gamma\right)$ reaction, is investigated. Structural information relevant to the population of states in these hypernuclei in recent $\left(e, e^{\prime} K^{+}\right)$studies is also given. Finally, the extension of the shell-model calculations to sd-shell hypernuclei is briefly considered.
\end{abstract}

Key words: Hypernuclei; Shell-model

PACS: 21.80.+n; 21.60.Cs

\section{Introduction}

This article provides an update on the shell-model interpretation of $\gamma$-ray transitions in p-shell hypernuclei [1] from a previous special issue on recent advances in strangeness nuclear physics and the start of an extension to sdshell hypernuclei. The experimental data available at the time was reviewed by Tamura in the same volume [2] and consisted of $22 \gamma$-ray transitions in ${ }_{\Lambda}^{7} \mathrm{Li}$, ${ }_{\Lambda}^{9} \mathrm{Be},{ }_{\Lambda}^{11} \mathrm{~B},{ }_{\Lambda}^{12} \mathrm{C},{ }_{\Lambda}^{13} \mathrm{C},{ }_{\Lambda}^{15} \mathrm{~N}$, and ${ }_{\Lambda}^{16} \mathrm{O}$, together with a limit on the ground-state doublet spacing in ${ }_{\Lambda}^{10} \mathrm{~B}$. Since then, new results on ${ }_{\Lambda}^{11} \mathrm{~B}$ and ${ }_{\Lambda}^{12} \mathrm{C}$ from KEK E566 using an upgraded germanium detector array, Hyperball2, have been reported at the Hyp-X conference by Tamura [3] and Ma [4. The groundstate doublet spacing in ${ }_{\Lambda}^{12} \mathrm{C}$ is established as $161 \mathrm{keV}$ both from the direct observation of the $161 \mathrm{keV} \gamma$-ray and from transitions from an excited $1^{-}$state

Email address: millener@bnl.gov (D.J. Millener). 
at $2832 \mathrm{keV}$. The ground-state doublet spacing is closely related to that of ${ }_{\Lambda}^{10} \mathrm{~B}$ which is $<100 \mathrm{keV}$. It seems that the difference between ${ }_{\Lambda}^{10} \mathrm{~B}$ and ${ }_{\Lambda}^{12} \mathrm{C}$ can be explained only by invoking the coupling between $\Lambda$ and $\Sigma$ hypernuclear states [5] $(\Lambda-\Sigma$ coupling).

Section 2 describes the shell model calculations and Section 3 summarizes the previously obtained results for transitions observed in the Hyperball experiments. Sections 4, 5, and 6 discuss the $A=8,9$, and 10 hypernuclei. Section 7 contains some information on contributions to the ground-state binding energies of p-shell hypernuclei. Section 8 is devoted to ${ }_{\Lambda}^{19} \mathrm{~F}$ while Section 9 contains a concluding discussion.

\section{Shell-model calculations}

Shell-model calculations for p-shell hypernuclei start with the Hamiltonian

$$
H=H_{N}+H_{Y}+V_{N Y}
$$

where $H_{N}$ is an empirical Hamiltonian for the p-shell core, the single-particle $H_{Y}$ supplies the $\sim 80 \mathrm{MeV}$ mass difference between $\Lambda$ and $\Sigma$, and $V_{N Y}$ is the $Y N$ interaction. The shell-model basis states are chosen to be of the form $\left|\left(p^{n} \alpha_{c} J_{c} T_{c}, j_{Y} t_{Y}\right) J T\right\rangle$, where the hyperon is coupled in angular momentum and isospin to eigenstates of the p-shell Hamiltonian for the core, with up to three values of $T_{c}$ contributing for $\Sigma$-hypernuclear states. This is known as a weak-coupling basis and, indeed, the mixing of basis states in the hypernuclear eigenstates is generally very small. In this basis, the core energies are taken from experiment where possible and from the p-shell calculation otherwise.

The $\Lambda N$ effective interaction can be written [6]

$$
V_{\Lambda N}(r)=V_{0}(r)+V_{\sigma}(r) \vec{s}_{N} \cdot \vec{s}_{\Lambda}+V_{\Lambda}(r) \vec{l}_{N \Lambda} \cdot \vec{s}_{\Lambda}+V_{\mathrm{N}}(r) \vec{l}_{N \Lambda} \cdot \vec{s}_{N}+V_{\mathrm{T}}(r) S_{12},
$$

where $S_{12}=3\left(\vec{\sigma}_{N} \cdot \vec{r}\right)\left(\vec{\sigma}_{\Lambda} \cdot \vec{r}\right)-\vec{\sigma}_{N} \cdot \vec{\sigma}_{\Lambda}$. The spin-orbit interactions can alternatively be expressed in terms of the symmetric (SLS) and antisymmetric (ALS) spin-orbit operators $\vec{l}_{N \Lambda} \cdot\left(\vec{s}_{\Lambda} \pm \vec{s}_{N}\right)$. The five $p_{N} s_{\Lambda}$ two-body matrix elements depend on the radial integrals associated with each component in Eq. (21), conventionally denoted by the parameters $\bar{V}, \Delta, S_{\Lambda}, S_{N}$ and $T$ [6]. By convention [6], $S_{\Lambda}$ and $S_{N}$ are actually the coefficients of $\vec{l}_{N} \cdot \vec{s}_{\Lambda}$ and $\vec{l}_{N} \cdot \vec{s}_{N}$. Then, the operators associated with $\Delta$ and $S_{\Lambda}$ are $\vec{S}_{N} \cdot \vec{s}_{\Lambda}$ and $\vec{L}_{N} \cdot \vec{s}_{\Lambda}$.

The parametrization of Eq. (2) applies to the direct $\Lambda N$ interaction, the $\Lambda N-$ $\Sigma N$ coupling interaction, and the direct $\Sigma N$ interaction for both isospin $1 / 2$ 
and 3/2. Values for the parameters based on various Nijmegen models of the $Y N$ interactions are given in Section 3 of Ref. [5]. Formally, one could include an overall factor $\sqrt{4 / 3} t_{N} \cdot t_{\Lambda \Sigma}$ in the analog of Eq. (2) that defines the interaction, where $t_{\Lambda \Sigma}$ is the operator that converts a $\Lambda$ into a $\Sigma$. Then, the core operator associated with $\bar{V}^{\prime}$ is $T_{N}=\sum_{i} t_{N i}$. This leads to a non-zero matrix element only between $\Lambda$ and $\Sigma$ states that have the same core, with the value

$$
\left\langle\left(J_{c} T, s_{\Sigma}\right) J T\left|V_{\Lambda \Sigma}^{\prime}\right|\left(J_{c} T, s_{\Lambda}\right) J T\right\rangle=\sqrt{4 / 3} \sqrt{T(T+1)} \bar{V}^{\prime}
$$

in analogy to Fermi $\beta$ decay of the core nucleus. Similarly, the spin-spin term involves $\sum_{i} s_{N i} t_{N i}$ for the core and connects core states that have large GamowTeller (GT) matrix elements between them. This point has been emphasized by Umeya and Harada [7] in a recent article on the effects of $\Lambda-\Sigma$ coupling in ${ }_{\Lambda}^{7-10} \mathrm{Li}$.

In an LS basis for the core, the matrix elements of $\vec{S}_{N} \cdot \vec{s}_{\Lambda}$ are diagonal (similarly

for $\left.\vec{L}_{N} \cdot \vec{s}_{\Lambda}=\left(\vec{J}_{N}-\vec{S}_{N}\right) \cdot \vec{s}_{\Lambda}\right)$ and depend just on the intensities of the total $L$ and $S$ for the hypernucleus. Because supermultiplet symmetry $\left[f_{c}\right] K_{c} L_{c} S_{c} J_{c} T_{c}$ is generally a good symmetry for p-shell core states, only one or two values of $L$ and $S$ are important. The mixing of different $\left[f_{c}\right] L_{c} S_{c}$ is primarily due to the vector (SLS plus ALS) terms in the p-shell Hamiltonian. Of the remaining $\Lambda N$ parameters, $\bar{V}$ contributes only to the overall binding energy; $S_{N}$ does not contribute to doublet splittings in the weak-coupling limit but a negative $S_{N}$ augments the nuclear spin-orbit interaction and contributes to the spacings between states based on different core states; in general, there are not simple expressions for the coefficients of $T$.

Many hypernuclear calculations have used the venerable Cohen and Kurath interactions [8]. Here, the p-shell interaction has been refined using the following strategy. The one-body spin-orbit splitting between the $p_{3 / 2}$ and $p_{1 / 2}$ orbits is fixed to give a good description of the light p-shell nuclei (say for $A \leq 9)$. The overall strength of the tensor interaction is also fixed, ultimately to produce the cancellation in ${ }^{14} \mathrm{C} \beta$ decay. The well-determined linear combinations of the central and vector p-shell interactions are then chosen by fitting the energies of a large number of states that are known to be dominantly pshell in character, including the large spin-orbit splitting at $A=15$. A detailed discussion of p-shell nuclei is given in Section 5 of Ref. [9].

\section{$3 \quad$ Measured doublet spacings}

Table 1 gives a summary of the contributions from $\Lambda-\Sigma$ coupling and the $\Lambda N$ interaction parameters to all 9 of the measured doublet spacings. Details, 
Table 1

Doublet spacings in p-shell hypernuclei. Energies are given in $\mathrm{keV}$. The entries in the top (bottom) half of the table are calculated using the parameters in Eq. (4) (Eq. (6) $)$. The individual contributions do not sum to exactly $\Delta E^{t h}$, which comes from the diagonalization, because small contributions from the energies of admixed core states are not included.

\begin{tabular}{|c|c|c|c|c|c|c|c|c|c|}
\hline & $J_{u}^{\pi}$ & $J_{l}^{\pi}$ & $\Lambda \Sigma$ & $\Delta$ & $S_{\Lambda}$ & $S_{N}$ & $T$ & $\Delta E^{t h}$ & $\Delta E^{e x p}$ \\
\hline${ }_{\Lambda}^{7} \mathrm{Li}$ & $3 / 2^{+}$ & $1 / 2^{+}$ & 72 & 628 & -1 & -4 & -9 & 693 & 692 \\
\hline${ }_{\Lambda}^{7} \mathrm{Li}$ & $7 / 2^{+}$ & $5 / 2^{+}$ & 74 & 557 & -32 & -8 & -71 & 494 & 471 \\
\hline${ }_{\Lambda}^{9} \mathrm{Be}$ & $3 / 2^{+}$ & $5 / 2^{+}$ & -8 & -14 & 37 & 0 & 28 & 44 & 43 \\
\hline${ }_{\Lambda}^{11} \mathrm{~B}$ & $7 / 2^{+}$ & $5 / 2^{+}$ & 56 & 339 & -37 & -10 & -80 & 267 & 264 \\
\hline${ }_{\Lambda}^{11} \mathrm{~B}$ & $3 / 2^{+}$ & $1 / 2^{+}$ & 61 & 424 & -3 & -44 & -10 & 475 & 505 \\
\hline${ }_{\Lambda}^{12} \mathrm{C}$ & $2^{-}$ & $1^{-}$ & 61 & 175 & -12 & -13 & -42 & 153 & 161 \\
\hline${ }_{\Lambda}^{15} \mathrm{~N}$ & $3 / 2_{2}^{+}$ & $1 / 2_{2}^{+}$ & 65 & 451 & -2 & -16 & -10 & 507 & 481 \\
\hline${ }_{\Lambda}^{16} \mathrm{O}$ & $1^{-}$ & $0^{-}$ & -33 & -123 & -20 & 1 & 188 & 23 & 26 \\
\hline${ }_{\Lambda}^{16} \mathrm{O}$ & $2^{-}$ & $1_{2}^{-}$ & 92 & 207 & -21 & 1 & -41 & 248 & 224 \\
\hline
\end{tabular}

such as figures showing spectra and tables giving breakdowns of energy-level spacings, wave functions, and transition rates, can be found in Refs. [19]. The set of parameters used for ${ }_{\Lambda}^{7} \mathrm{Li}$ and ${ }_{\Lambda}^{9} \mathrm{Be}$ (chosen to fit the energy spacings in ${ }_{\Lambda}^{7} \mathrm{Li}$ ) is (parameters in $\mathrm{MeV}$ )

$$
\Delta=0.430 \quad S_{\Lambda}=-0.015 \quad S_{N}=-0.390 \quad T=0.030 .
$$

The matrix elements for the $\Lambda-\Sigma$ coupling interaction, based on the G-matrix calculations of Ref. [10] for the nsc97e, $f$ interactions [11], are [1]9]

$$
\bar{V}^{\prime}=1.45 \quad \Delta^{\prime}=3.04 \quad S_{\Lambda}^{\prime}=S_{N}^{\prime}=-0.09 \quad T^{\prime}=0.16
$$

These parameters are kept fixed throughout the p-shell in the present calculations.

The ground-state doublet in ${ }_{\Lambda}^{7} \mathrm{Li}$ and the exited-state doublets in ${ }_{\Lambda}^{11} \mathrm{~B}$ and ${ }_{\Lambda}^{15} \mathrm{~N}$ are based on core states that are largely $L_{c}=0$ and $S_{c}=1$. This limits contributions from other than $\Delta$, which enters with a large coefficient of $3 / 2$ in the $L_{c}=0$ limit, and $\Lambda-\Sigma$ coupling. The coefficient of $\Delta$ for the excited state doublet is $7 / 6$ for $L_{c}=2, S_{c}=1, J_{c}=3$ but here $S_{\Lambda}$ and $T$ enter with substantial coefficients. The $3^{+}$core state is the lowest member of an $L_{c}=2$, $S_{c}=1$ triplet and moves down in energy if the strength of the nuclear spin- 
orbit interaction is increased, as it is by $S_{N}$, in the hypernucleus. The value of $S_{N}$ in Eq. (4) is chosen to reproduce the $2.05-\mathrm{MeV}$ excitation energy of the $5 / 2^{+}$state in ${ }_{\Lambda}^{7} \mathrm{Li}$.

The ${ }^{8}$ Be core state for the ${ }_{\Lambda}^{9}$ Be doublet has mainly $L_{c}=2$ and $S_{c}=0$, giving a coefficient of $-5 / 2$ for $S_{\Lambda}$. The contributions from $\Delta$ and $T$, arising from small $S_{c}=1$ components, together with the small $\Lambda-\Sigma$ coupling contribution, happen to more or less cancel. This means that the ${ }_{\Lambda}^{9}$ Be doublet spacing demands a small value for $S_{\Lambda}$.

The doublet spacings for the heavier p-shell hypernuclei consistently require a smaller value for $\Delta$

$$
\Delta=0.330 \quad S_{\Lambda}=-0.015 \quad S_{N}=-0.350 \quad T=0.0239
$$

$T$ plays a particularly important role in the ground-state doublet $\left(p_{1 / 2}^{-1} s_{\Lambda}\right)$ splitting of ${ }_{\Lambda}^{16} \mathrm{O}$ and is determined from a measurement of the doublet spacing [12,13. The ground-state doublet spacing of ${ }_{\Lambda}^{15} \mathrm{~N}$, which is closely related to that of ${ }_{\Lambda}^{16} \mathrm{O}$, is missing form Table 1 because the transition to the $1 / 2^{+}$ state from the $2268-\mathrm{keV} 1 / 2^{+} ; 1$ level is not observed due to a subtle cancellation [1,9]. However, mesonic weak-decay studies have determined that the ground-state spin-parity of ${ }_{\Lambda}^{15} \mathrm{~N}$ is $3 / 2^{+}$[14,15. In Eq. (6) $), S_{N}$ fits the increase in the excitation energy of the excited-state doublet over the spacing of the p-hole states in ${ }^{15} \mathrm{O}$.

As can be seen from Table 1, there is a consistent description of the doublet spacings once a larger value of $\Delta$ is taken for ${ }_{\Lambda}^{7} \mathrm{Li}$. A conjecture, as yet unproven, is that shell-model admixtures beyond $0 \hbar \omega$ for the lightest p-shell nuclei $\left({ }^{6} \mathrm{Li}\right.$ in particular) involve mainly excitations from the s-shell to the pshell, thus permitting an active role for $s_{N} s_{\Lambda}$ matrix elements that are larger than those for $p_{N} s_{\Lambda}$. For $A=10$ and beyond, higher admixtures involve $p \rightarrow s d$ excitations and bring in smaller $\Lambda N$ matrix elements.

Finally, it is clear [1,5,9] that a term such as $S_{N}$ is necessary to describe the spacings between states based on different core states. Formally, the $S_{N}$ term arises from a combination of the SLS and ALS interactions but, in practice, $S_{N}$ is treated as a fitting parameter. A two-body $N N$ ALS interaction that gives rise to similar effects comes from the double one-pion exchange $\Lambda N N$ interaction averaged over the $s_{\Lambda}$ wave function, as in the original work of Gal, Soper, and Dalitz [6]. While a one-body $S_{N}$ term appears to be adequate near the beginning and end of the p-shell, there is a need for a much larger effect for (at least) ${ }_{\Lambda}^{11} \mathrm{~B},{ }_{\Lambda}^{12} \mathrm{C}$, and ${ }_{\Lambda}^{13} \mathrm{C}$. The high excitation energy (1483 keV) of the first $1 / 2^{+}$state in ${ }_{\Lambda}^{11} \mathrm{~B}$, taken together with the known spacings of the ground-state and first-excited state doublets, means that the spacing of the doublet centroids is $1669 \mathrm{keV}$ compared with the $1^{+} / 3^{+}$core separation of 


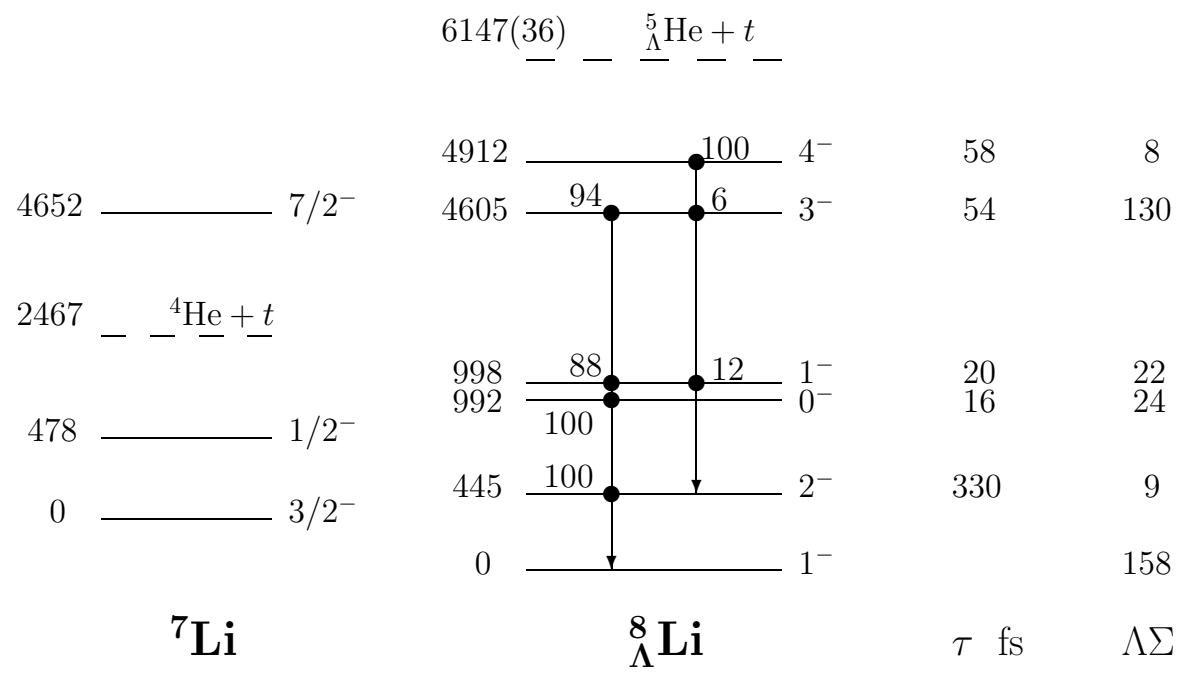

Fig. 1. The spectrum of ${ }_{\Lambda}^{8} \mathrm{Li}$. The ${ }^{7} \mathrm{Li}$ core states are shown on the left. The $\gamma$-ray branching ratios and lifetimes are theoretical. For each state of ${ }_{\Lambda}^{8} \mathrm{Li}$, the calculated energy shifts due to $\Lambda-\Sigma$ coupling are given. All energies are in $\mathrm{keV}$.

$718 \mathrm{keV}$. However, the $S_{N}$ value of Eq. (6) gives just over $400 \mathrm{keV}$ towards the difference. While there is sensitivity to the core wave functions, the high excitation energies of $2832 \mathrm{keV}$ for the $1_{2}^{-}$state of ${ }_{\Lambda}^{12} \mathrm{C}$ and $4880 \mathrm{keV}$ for the $3 / 2_{1}^{+}$state in ${ }_{\Lambda}^{13} \mathrm{C}$ cannot be explained either with this value of $S_{N}$.

\section{The $A=8$ hypernuclei}

Figure 1 gives a theoretical spectrum, including $\gamma$-ray branching ratios and lifetimes, for ${ }_{\Lambda}^{8} \mathrm{Li}$. Because the lowest $3 / 2^{-}$and $1 / 2^{-}$states of ${ }^{7} \mathrm{Li}$ are closely spaced and both have $L_{c}=1$, the bound $1^{-}$states of ${ }_{\Lambda}^{8} \mathrm{Li}$ involve significant mixing $\left(\left|1_{1}^{-}\right\rangle=0.946\left(3 / 2^{-}\right) \times s_{\Lambda}-0.319\left(1 / 2^{-}\right) \times s_{\Lambda}\right)$ of the configurations based on them. On a historical note, the spin-parity of ${ }_{\Lambda}^{8} \mathrm{Li}$ [16] and restrictions on the mixing [17] were derived from emulsion data on the decay ${ }_{\Lambda}^{8} \mathrm{Li} \rightarrow \pi^{-}$ $+{ }^{8} \mathrm{Be}^{*} \rightarrow \pi^{-}+\alpha+\alpha$. The next largest admixtures are actually from the corresponding $\Sigma$-hypernuclear states.

As can be see from Fig. 1, the energy shift due $\Lambda$ - $\Sigma$ coupling for the $1^{-}$ ground state is large in strong contrast to that for the $2^{-}$member of the doublet. In fact, $\Lambda-\Sigma$ coupling is predicted to account for a third of the doublet spacing. The predicted energy spacing is very close to the $442.1(21) \mathrm{keV}$ energy of a $\gamma$-ray observed following the production of highly-excited states of ${ }_{\Lambda}^{10} \mathrm{~B}$ via the $\left(K^{-}, \pi^{-}\right)$reaction [18]. The $442 \mathrm{keV} \gamma$-ray was tentatively attributed to ${ }_{\Lambda}^{8} \mathrm{Li}$ or its mirror hypernucleus ${ }_{\Lambda}^{8} \mathrm{Be}\left[18\right.$, the $2^{-}$state of which could be reached by $l=1$ deuteron emission from the $s^{4} p^{4}(s d) s_{\Lambda}$ component of the $3^{+} s_{n}^{-1} s_{\Lambda}$ substitutional state in ${ }_{\Lambda}^{10} \mathrm{~B}$ (the excited-state doublet in ${ }_{\Lambda}^{7} \mathrm{Li}$ was 
studied following $l=0{ }^{3} \mathrm{He}$ emission from the same state [19]). If confirmed, the 442-keV $\gamma$-ray would provide additional strong support for the important role played by $\Lambda-\Sigma$ coupling in hypernuclear spectra.

A $1.22(4)-\mathrm{MeV} \gamma$-ray, seen after $K^{-}$mesons were stopped in a ${ }^{9} \mathrm{Be}$ target [20], was tentatively ascribed to ${ }_{\Lambda}^{8} \mathrm{Li}$, possibly as a transition between the $1^{-}$states in Fig. 1. In Ref. [21], it was found to be difficult to explain such a high energy. This is still the case despite the addition of $136 \mathrm{keV}$ to the transition energy from $\Lambda-\Sigma$ coupling.

The role of $\Lambda-\Sigma$ coupling for the ground-state doublet and the excited $1^{-}$ state can be seen from the interplay of $\bar{V}^{\prime}$ and $\Delta^{\prime}$ in the coupling matrix elements and the use of perturbation theory for the energy shifts $\left(\sim v^{2} / \Delta E\right.$ with $\Delta E \sim 80 \mathrm{MeV}$ ). From Eq. (5), $\bar{V}^{\prime}$ gives a contribution of $1.45 \mathrm{MeV}$ to the diagonal coupling matrix elements involving the same core state. Adding the remaining $\Lambda-\Sigma$ contributions (mainly from $\Delta^{\prime}$ ) for the $3 / 2^{-}$core states gives $2.6524 \mathrm{MeV}$ for $J^{\pi}=1^{-}$and $0.7286 \mathrm{MeV}$ for $J^{\pi}=2^{-}$. The off-diagonal matrix elements (from $\Delta^{\prime}$ ) involving $1 / 2^{-}$and $3 / 2^{-}$core states are both $-1.5094 \mathrm{MeV}$, while the diagonal $1 / 2^{-}$matrix element is $1.4761 \mathrm{MeV}$. In the weak-coupling limit, the push on the ground state is $88 \mathrm{keV}$ from the $\left(3 / 2^{-} \times s_{\Sigma}\right)$ state and $28 \mathrm{keV}$ from the $\left(1 / 2^{-} \times s_{\Sigma}\right)$ state while the push on the $2^{-}$state is only 7 $\mathrm{keV}$. The mixing between the $\left(3 / 2^{-} \times s_{\Lambda}\right)$ and $\left(1 / 2^{-} \times s_{\Lambda}\right)$ states increases the $\Lambda-\Sigma$ coupling matrix elements for the lower $1^{-}$state and decreases them for the upper state; putting in the numbers from above, an estimate of $157 \mathrm{keV}$ is obtained for the push on the ground state.

As far as electromagnetic transitions are concerned, the p-shell wave functions account well for the M1 properties of the $3 / 2^{-}$and $1 / 2^{-}$core states using the bare M1 operator, leaving room for the expected small enhancement of the isovector matrix elements by meson-exchange currents 22]; the calculated magnetic moments of ${ }^{7} \mathrm{Li}$ and ${ }^{7} \mathrm{Be}$ are $3.145 \mu_{N}$ and $-1.263 \mu_{N}$ compared with the experimental values of $3.256 \mu_{N}$ and $-1.399 \mu_{N}$, respectively. Because the M1 matrix element for the ground-state doublet transition is proportional to $g_{c}-g_{\Lambda}\left(g_{\Lambda}=-1.226\right)$ in the weak-coupling limit [23] the transition is going to be much faster in the odd-proton nucleus ${ }_{\Lambda}^{8} \mathrm{Li}$. This simple approximation does not apply because of the configuration mixing in the $1^{-}$wave functions. The M1 transition strengths end up being 1.086 W.u. in ${ }_{\Lambda}^{8} \mathrm{Li}$ and $0.043 \mathrm{~W} . \mathrm{u}$. in ${ }_{\Lambda}^{8} \mathrm{Be}(\tau=8.37 \mathrm{ps})$. For the decay of the $1_{2}^{-}$state in ${ }_{\Lambda}^{8} \mathrm{Be}$, one obtains a branch of $64 \%$ to the ground state and a lifetime of 46 fs. All these low-energy transitions are little affected by their E2 components.

As a further example of the effects of $\Lambda-\Sigma$ coupling, the ${ }_{\Lambda}^{8} \mathrm{He}$ ground-state doublet is predicted to have a spacing of $101 \mathrm{keV}$ with $\Lambda-\Sigma$ contributions of 154 and $182 \mathrm{keV}$ to the binding energies of the $1^{-}$and $2^{-}$states, respectively. In this case, $\Lambda-\Sigma$ coupling reduces the doublet spacing by $28 \mathrm{keV}$. 


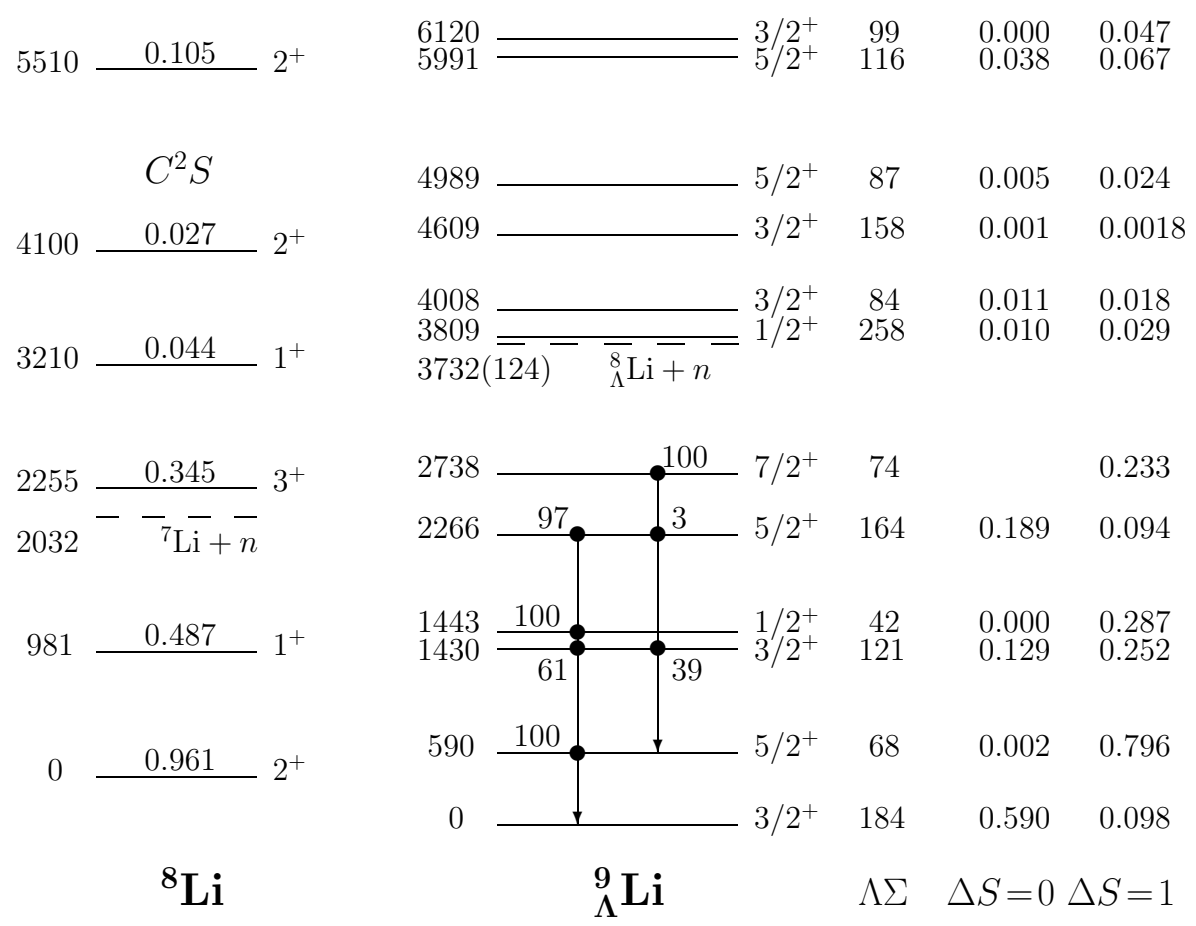

Fig. 2. The spectrum of ${ }_{\Lambda}^{9} \mathrm{Li}$. The ${ }^{8} \mathrm{Li}$ core states are shown on the left along with the spectroscopic factors for proton removal from ${ }^{9} \mathrm{Be}$. The $\gamma$-ray branching ratios and lifetimes are theoretical. For each state of ${ }_{\Lambda}^{9} \mathrm{Li}$, the calculated energy shifts due to $\Lambda-\Sigma$ coupling are given. All energies are in $\mathrm{keV}$. On the right, the structural factors (defined in Appendix A) giving the relative population of levels in purely non-spin-flip $(\Delta S=0)$ and purely spin-flip $(\Delta S=1)$ production reactions on a ${ }^{9} \mathrm{Be}$ target are given.

\section{The ${ }_{\Lambda}^{9} \mathrm{Li}$ hypernucleus}

There is interest in ${ }_{\Lambda}^{9} \mathrm{Li}$ because it has been studied using the ${ }^{9} \mathrm{Be}\left(e, e^{\prime} K^{+}\right){ }_{\Lambda}^{9} \mathrm{Li}$ reaction at JLab [24] and could be studied via the ${ }^{9} \mathrm{Be}\left(K^{-}, \pi^{0} \gamma\right)_{\Lambda}^{9} \mathrm{Li}$ reaction at J-PARC. In addition, it is the possible source of a $1303-\mathrm{keV} \gamma$-ray seen in a stopped $K^{-}$experiment [25], most strongly on a ${ }^{9}$ Be target.

Figure 2 gives a theoretical spectrum, including $\gamma$-ray branching ratios and lifetimes, for ${ }_{\Lambda}^{9} \mathrm{Li}$. Because non-spin-flip production is dominant in the $\left(K^{-}, \pi^{0}\right)$ reaction at rest, the only likely candidate for the $1303-\mathrm{keV} \gamma$-ray is the excited $3 / 2^{+}$to ground state transition. In this case, an $840-\mathrm{keV}$ transition to the lowest $5 / 2^{+}$state should also be observable. The predicted energy of 1430 $\mathrm{keV}$ is too high. For comparison, the $\Lambda N$ parameter set in Eq. (6) predicts the $3 / 2_{2}^{+}$state at $1331 \mathrm{keV}$ and the $5 / 2_{1}^{+}$state at $471 \mathrm{keV}$. It is clear that an in-flight $\left(K^{-}, \pi^{0} \gamma\right)$ study with the Hyperball-J at an incident $K^{-}$energy where spin-flip amplitudes are important is desirable.

From the ${ }^{9} \mathrm{Be}(t, \alpha)^{8} \mathrm{Li}$ study by Liu and Fortune [26] and the pickup spectro- 
scopic factors given in Fig. 2, the bulk of the cross section for the production of $s_{\Lambda}$ states in the ${ }^{9} \mathrm{Be}\left(e, e^{\prime} K^{+}\right){ }_{\Lambda}^{9} \mathrm{Li}$ reaction is expected to be concentrated in states built on the lowest three states of ${ }^{8} \mathrm{Li}$ (see Ref. [27] for an early theoretical study). This is indeed the case [24]. The strongest observed state is the upper member of the ground-state doublet but there appears to be more strength in the states based on the $3^{+}$core state than predicted and a disagreement about the location of the strength based on the $1^{+}$state. The strength based on the $1_{2}^{+}$state is close enough to the neutron threshold that the states should be narrow and any significant strength associated with them should be observable in the electro-production reaction. This would be the case for the (8-16)2BME and (8-16)POT interactions of Cohen and Kurath [8] but not for the (6-16)2BME interactions or the various fitted interactions used in recent hypernuclear studies. The former interactions favor the second $1^{+}$ state in proton removal from ${ }^{9}$ Be because the lowest $1^{+}$state is dominantly $L_{c}=1, S_{c}=1$ rather than strongly mixed $S_{c}=0$ and $S_{c}=1$. The use of the (8-16)2BME interaction is the reason that the ${ }_{\Lambda}^{9} \mathrm{Li}$ spectrum of Umeya and Harada [7] looks rather different from the one in Fig. 2,

\section{The $A=10$ hypernuclei}

Figure 3 gives a theoretical spectrum, including $\gamma$-ray branching ratios, pickup spectroscopic factors, and formation factors for ${ }_{\Lambda}^{10} \mathrm{Be}$.

${ }_{\Lambda}^{10} \mathrm{Be}$ is another hypernucleus that could be studied via the $\left(K^{-}, \pi^{0} \gamma\right)$ reaction with the Hyperball-J at J-PARC, this time with a ${ }^{10} \mathrm{~B}$ target. A strong reason for doing so would be to try to measure the ground-state doublet spacing by observing transitions to both members from a higher level. The obvious candidate is the $2^{-}$level based on the $5 / 2^{-}$core level of ${ }^{9} \mathrm{Be}$. Unfortunately, the $2^{-} \rightarrow 2^{-}$transition is strongly hindered with respect to the $2^{-} \rightarrow 1^{-}$ transition by a factor of 15 from the recoupling coefficient (but gains something back on the $2 J_{f}+1$ factor). In the weak-coupling limit, the $2^{-} \rightarrow 2^{-}$branch would be only $9 \%$ but something is gained from configuration mixing. Again, the E2 components of the transitions are not very important.

The spacings of the ground-state and excited-state doublets are predicted to be very similar. This could certainly be checked in a $\left(K^{-}, \pi^{0} \gamma\right)$ experiment and which $\gamma$-ray is which could be determined by choosing $K^{-}$momenta for which the ratio of spin-flip to non-spin-flip is quite different. The $1^{-}$level based on the broad $1 / 2^{-}$level in ${ }^{9}$ Be could be populated via the $\left(K^{-}, \pi^{-}\right)$reaction on ${ }^{10} \mathrm{Be}$ if only a thick enough ${ }^{10} \mathrm{Be}$ target could be made.

As far as production reactions are concerned, the ${ }^{10} \mathrm{~B}\left(K^{-}, \pi^{-}\right){ }_{\Lambda}^{10} \mathrm{~B}$ reaction has been studied in KEK E336 [28]. From the spectroscopic factors for proton 


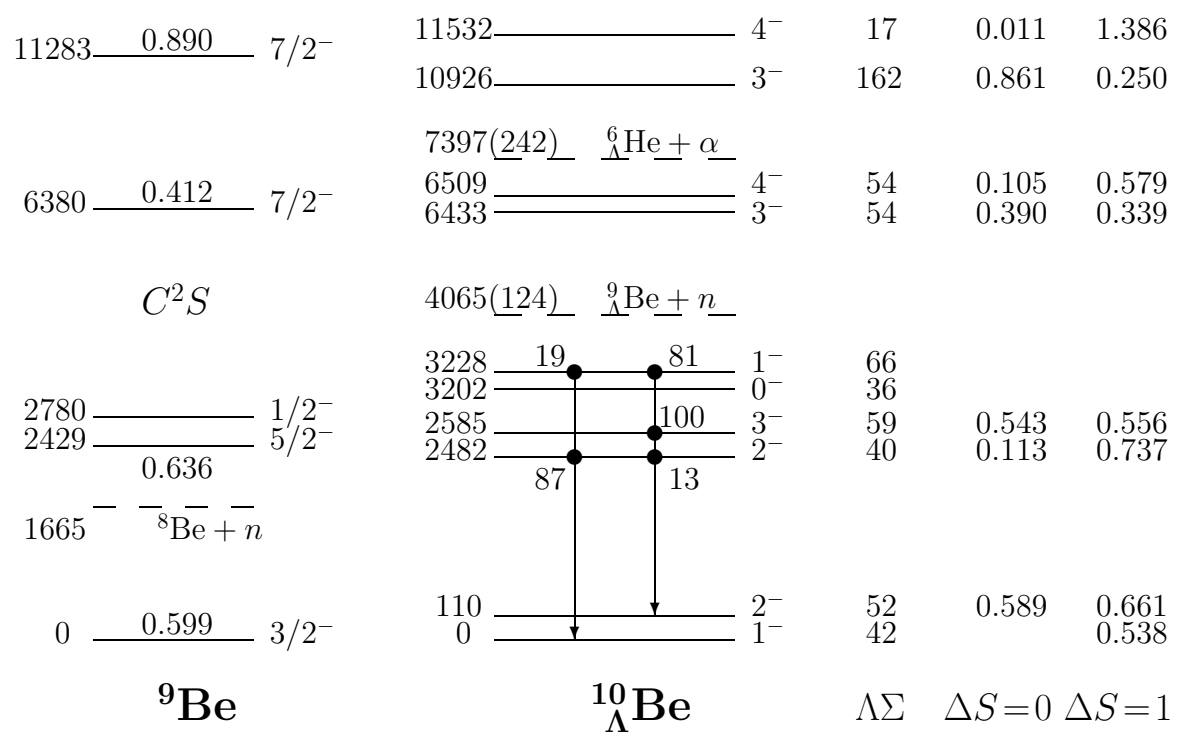

Fig. 3. The spectrum of ${ }_{\Lambda}^{10} \mathrm{Be}$. The ${ }^{9}$ Be core states are shown on the left along with the spectroscopic factors for proton removal from ${ }^{10} \mathrm{~B}$. The $\gamma$-ray branching ratios and lifetimes are theoretical. For each state of ${ }_{\Lambda}^{10} \mathrm{Be}$, the calculated energy shifts due to $\Lambda-\Sigma$ coupling are given. All energies are in keV. On the right, the structural factors giving the relative population of levels in purely non-spin-flip $(\Delta S=0)$ and purely spin-flip $(\Delta S=1)$ production reactions on a ${ }^{10} \mathrm{~B}$ target are given.

or neutron removal from ${ }^{10} \mathrm{~B}$, one expects to see four strong peaks up to about $10 \mathrm{MeV}$ in excitation energy. In the $\left(K^{-}, \pi^{-}\right)$experiment, these are not cleanly resolved but the data has been fitted to extract energies and relative yields [28]. The relative yields are in good agreement with the $\Delta S=0$ structure factors in Fig. 3 but the spectrum is somewhat expanded with respect to that extracted from the data (the extracted energy may be affected by the steeply rising background that extends under the fourth peak). A spectrum in which the four peaks, based on the core states reached strongly by proton removal from ${ }^{10} \mathrm{~B}$, are cleanly separated has recently been obtained using the ${ }^{10} \mathrm{~B}\left(e, e^{\prime} K^{+}\right){ }_{\Lambda}^{10} \mathrm{Be}$ reaction at JLab [29]. This is as predicted by Motoba et al. [30] and by the results in Fig. 3 based on more recent information on inmedium $Y N$ interactions. States involving a $p_{\Lambda}$ coupled to the same core states are also expected to be strongly populated (again see Ref. [30]) and it will be interesting to make a detailed comparison between theory and experiment.

\section{Contributions to $\Lambda$ binding energies}

Table 2 shows the $\Lambda-\Sigma$ and spin-dependent contributions to the ground-state binding energies for a wide range of p-shell hypernuclei. The sum of these contributions can reach $1 \mathrm{MeV}$. The experimental $B_{\Lambda}$ values are from emulsion 
Table 2

$\Lambda-\Sigma$ and spin-dependent contributions to ground-state binding energies (in $\mathrm{keV}$ ). The units for $B_{\Lambda}$ and $\bar{V}$ in the last two columns are MeV. The experimental $B_{\Lambda}$ values and errors are taken from Ref. [31. No $\bar{V}$ is given for ${ }_{\Lambda}^{9} \mathrm{Be}$ which is a special case because of the unbound nature of ${ }^{8} \mathrm{Be}$, the binding energy of which enters into $B_{\Lambda}\left({ }_{\Lambda}^{9} \mathrm{Be}\right)$. The entry for ${ }_{\Lambda}^{12} \mathrm{Be}$ is based on p-shell core states but the ground state could have positive parity.

\begin{tabular}{|c|c|c|c|c|c|c|c|c|c|}
\hline & $J^{\pi}$ & $\Lambda-\Sigma$ & $\Delta$ & $S_{\Lambda}$ & $S_{N}$ & $T$ & Sum & $B_{\Lambda}^{e x p t}(\Delta B)$ & $\bar{V}$ \\
\hline${ }_{\Lambda}^{7} \mathrm{He}$ & $1 / 2^{+}$ & 101 & 1 & 0 & 176 & 0 & 278 & & \\
\hline${ }_{\Lambda}^{8} \mathrm{He}$ & $1^{-}$ & 154 & 152 & -13 & 454 & -38 & 709 & $7.16(70)$ & -1.11 \\
\hline${ }_{\Lambda}^{9} \mathrm{He}$ & $1 / 2^{+}$ & 253 & 6 & 0 & 619 & 1 & 879 & & \\
\hline${ }_{\Lambda}^{7} \mathrm{Li}$ & $1 / 2^{+}$ & 78 & 419 & 0 & 94 & -2 & 589 & $5.58(3)$ & -0.94 \\
\hline${ }_{\Lambda}^{8} \mathrm{Li}$ & $1^{-}$ & 160 & 288 & -6 & 192 & -9 & 625 & $6.80(3)$ & -1.02 \\
\hline${ }_{\Lambda}^{9} \mathrm{Li}$ & $3 / 2^{+}$ & 183 & 350 & -10 & 434 & -6 & 952 & $8.50(12)$ & -1.06 \\
\hline${ }_{\Lambda}^{10} \mathrm{Li}$ & $1^{-}$ & 275 & 175 & -11 & 595 & -12 & 1022 & & \\
\hline${ }_{\Lambda}^{9} \mathrm{Be}$ & $1 / 2^{+}$ & 4 & 0 & 0 & 207 & 0 & 211 & $6.71(4)$ & \\
\hline${ }_{\Lambda}^{11} \mathrm{Be}$ & $1 / 2^{+}$ & 99 & 2 & 0 & 540 & 0 & 641 & & \\
\hline${ }_{\Lambda}^{12} \mathrm{Be}$ & $0^{-}$ & 158 & -76 & -15 & 554 & 127 & 748 & & \\
\hline${ }_{\Lambda}^{10} \mathrm{~B}$ & $1^{-}$ & 35 & 125 & -13 & 386 & -15 & 518 & $8.89(12)$ & -1.05 \\
\hline${ }_{\Lambda}^{11} \mathrm{~B}$ & $5 / 2^{+}$ & 66 & 203 & -20 & 652 & -43 & 858 & $10.24(5)$ & -1.04 \\
\hline${ }_{\Lambda}^{12} \mathrm{~B}$ & $1^{-}$ & 103 & 108 & -14 & 704 & -29 & 869 & $11.37(6)$ & -1.05 \\
\hline${ }_{\Lambda}^{13} \mathrm{~B}$ & $1 / 2^{+}$ & 130 & 197 & -6 & 621 & -57 & 885 & & \\
\hline${ }_{\Lambda}^{14} \mathrm{~B}$ & $1^{-}$ & 255 & 115 & -13 & 458 & -30 & 785 & & \\
\hline${ }_{\Lambda}^{13} \mathrm{C}$ & $1 / 2^{+}$ & 28 & -4 & 0 & 841 & -1 & 864 & $11.69(12)$ & -0.96 \\
\hline${ }_{\Lambda}^{14} \mathrm{C}$ & $1^{-}$ & 75 & 47 & 6 & 816 & -40 & 904 & $12.17(33)$ & -0.91 \\
\hline${ }_{\Lambda}^{15} \mathrm{C}$ & $1 / 2^{+}$ & 116 & 8 & 0 & 636 & 2 & 762 & & \\
\hline${ }_{\Lambda}^{15} \mathrm{~N}$ & $3 / 2^{+}$ & 59 & 40 & 12 & 630 & -69 & 726 & $13.59(15)$ & -0.97 \\
\hline${ }_{\Lambda}^{16} \mathrm{~N}$ & $1^{-}$ & 62 & 94 & 6 & 349 & -45 & 412 & $13.76(16)$ & -0.93 \\
\hline
\end{tabular}

studies [31] (a number of mirror hypernuclei are not listed) except for ${ }_{\Lambda}^{16} \mathrm{~N}$ where the value comes from a study using the ${ }^{16} \mathrm{O}\left(e, e^{\prime} K^{+}\right){ }_{\Lambda}^{16} \mathrm{~N}$ reaction [32] (the observed $1^{-}$state is actually $26 \mathrm{keV}$ above the $0^{-}$ground state [12,13]). The remaining hypernuclei that are listed are chosen because the neutronrich p-shell cores have higher isospin and can exhibit larger effects from $\Lambda-\Sigma$ coupling. 
The spin-independent central component $\bar{V}$ of the $\Lambda N$ interaction doesn't affect the spectra but can be estimated from the binding energies by taking $B_{\Lambda}\left({ }_{\Lambda}^{5} \mathrm{He}\right)=3.12 \mathrm{MeV}$ as the $s_{\Lambda}$ single-particle energy and using

$$
B_{\Lambda}=3.12-n \bar{V}+\mathrm{Sum}
$$

where $n$ is the number of p-shell nucleons in the core. The values of $\bar{V}$ so extracted are given in the last column of Table 2 . The $\bar{V}$ are relatively constant and close to the values derived from $\Lambda N$ potential models [5]. In reality, small repulsive contributions quadratic in $n$ are expected from the double one-pion exchange interaction [6] which would then call for a somewhat more attractive $\bar{V}$. Quite good estimates can be made for $B_{\Lambda}$ values. In the case of $\Lambda \Lambda$ hypernuclei, two spin-averaged $B_{\Lambda}$ values enter into the binding energy along with a $\Lambda \Lambda$ two-body matrix element that is known to be quite small $(-0.67 \mathrm{MeV})$. Then, it is clear that the knowledge of single- $\Lambda$ binding energies can be used to make reliable estimates for the binding energies of $\Lambda \Lambda$ hypernuclei [33].

\section{8 sd-shell hypernuclei}

An extension of the studies of $\gamma$-ray transitions in p-shell hypernuclei is planned for ${ }_{\Lambda}^{19} \mathrm{~F}$ in J-PARC E13 [34]. The reason for choosing ${ }^{19} \mathrm{~F}$ as a target is that ${ }^{18} \mathrm{~F}$ has a primarily $L=0, S=1$ ground state so that one should observe a relatively large ground-state doublet spacing for ${ }_{\Lambda}^{19} \mathrm{~F}$, in complete analogy to ${ }_{\Lambda}^{7} \mathrm{Li}$ (and the ${ }_{\Lambda}^{15} \mathrm{~N}$ doublet based on the second $1^{+}$state in ${ }^{15} \mathrm{~N}$ ); ${ }^{19} \mathrm{~F}$ itself has a primarily $L=0, S=1 / 2$ ground state, which is why an (impractical) ${ }^{20} \mathrm{Ne}$ target was considered by Millener et al. [21].

The ${ }^{18} \mathrm{~F}$ core nucleus has quite a dense spectrum, including $3^{+} ; 0,0^{+} ; 1,0^{-} ; 0$, and $5^{+} ; 0$ states close to $1 \mathrm{MeV}$ (see Fig.3 in Ref. [34]). The wave functions for the lowest $1^{+}$and $3^{+}$states are given in a $j j$-coupling basis in Table 3 . The $1^{+}$state is actually $92.7 \% L=0, S=1$ (amplitudes 0.8985 and -0.3461 for SU(3) symmetry (40) and (02), respectively), while the $3^{+}$state is $96.9 \%$ $L=2, S=1$ (amplitudes 0.9722 and -0.1548). Historically, this simplicity for ${ }^{18} \mathrm{~F}$ and ${ }^{19} \mathrm{~F}$ was a significant factor in the introduction of Elliott's $\mathrm{SU}(3)$ model [36].

In the sd-shell, there are $8(s d)_{N} s_{\Lambda}$ matrix elements; 4 central, one each for LS and ALS in relative p states, and 2 tensor (in both even and odd states). These are shown as a function of binding energy in Table 4. Here, a radial representation of the $\Lambda N$ interaction that reproduces the matrix elements of Eq.(6) is used (cf. Ref. [5]). Table 4 demonstrates that the matrix elements are sensitive to the binding energies of the sd-shell orbits, especially the noded 
Table 3

${ }^{18} \mathrm{~F}$ wave functions using the Chung-Wildenthal interaction [35].

\begin{tabular}{rrrrrrr}
\hline$J^{\pi}$ & $d_{5 / 2}^{2}$ & $d_{5 / 2} d_{3 / 2}$ & $d_{3 / 2}^{2}$ & $d_{5 / 2} s_{1 / 2}$ & $d_{3 / 2} s_{1 / 2}$ & $s_{1 / 2}^{2}$ \\
\hline $1^{+}$ & 0.6038 & -0.6539 & -0.0515 & & 0.1130 & 0.4386 \\
$3^{+}$ & 0.5721 & -0.2397 & -0.0026 & 0.7844 & & \\
\hline
\end{tabular}

Table 4

$(s d)_{N} s_{\Lambda}$ matrix elements for harmonic oscillator (HO) and Woods-Saxon (WS) wave functions. The $s_{\Lambda}$ orbit is bound at $15 \mathrm{MeV}$. The sd orbits are bound as indicated, where Exp. means binding energies of 8, 4, and $9 \mathrm{MeV}$ for the $1 s_{1 / 2}$, $0 d_{3 / 2}$, and $0 d_{5 / 2}$ neutron orbits, taking into account that the neutron separation energy is $9.15 \mathrm{MeV}$ for ${ }^{18} \mathrm{~F}$. The well geometry has $r_{n}=1.26 \mathrm{fm}$ and $a_{n}=0.65 \mathrm{fm}$ for the neutron $(1.212 \mathrm{fm}$ and $0.60 \mathrm{fm}$ for the $\Lambda$ ).

\begin{tabular}{|c|c|c|c|c|c|}
\hline & & $\mathrm{HO}$ & WS & WS & WS \\
\hline & $J$ & $b=1.741 \mathrm{fm}$ & $\mathrm{BE}=\mathrm{Exp}$ & $\mathrm{BE}=9 \mathrm{MeV}$ & $\mathrm{BE}=1 \mathrm{MeV}$ \\
\hline \multirow[t]{2}{*}{$\left\langle 1 s_{1 / 2} s_{\Lambda}|V| 1 s_{1 / 2} s_{\Lambda}\right\rangle$} & 0 & -1.6067 & -1.2774 & -1.3181 & -0.6529 \\
\hline & 1 & -1.1817 & -0.9524 & -0.9822 & -0.4915 \\
\hline$\left\langle 0 d_{3 / 2} s_{\Lambda}|V| 1 s_{1 / 2} s_{\Lambda}\right\rangle$ & 1 & -0.1254 & -0.1062 & -0.1174 & -0.0610 \\
\hline \multirow[t]{2}{*}{$\left\langle 0 d_{3 / 2} s_{\Lambda}|V| 0 d_{3 / 2} s_{\Lambda}\right\rangle$} & 1 & -0.4883 & -0.4890 & -0.5522 & -0.3828 \\
\hline & 2 & -0.5184 & -0.5107 & -0.5747 & -0.4033 \\
\hline$\left\langle 0 d_{5 / 2} s_{\Lambda}|V| 0 d_{3 / 2} s_{\Lambda}\right\rangle$ & 2 & 0.1301 & 0.1333 & 0.1459 & 0.0936 \\
\hline \multirow[t]{2}{*}{$\left\langle 0 d_{5 / 2} s_{\Lambda}|V| 0 d_{5 / 2} s_{\Lambda}\right\rangle$} & 2 & -1.0508 & -1.0708 & -1.1026 & -0.7453 \\
\hline & 3 & -0.9863 & -1.0009 & -1.0309 & -0.6958 \\
\hline
\end{tabular}

$1 s$ orbit. We note that sd-shell orbits indeed become loosely bound and the $1 s$ orbit moves below the $0 d_{5 / 2}$ orbit for states in p-shell hypernuclei.

Combining the Woods-Saxon matrix elements for the Exp. case in Table 4 with the wave functions in Table [3, the doublet spacings for states based on the lowest $1^{+}$and $3^{+}$states are $305 \mathrm{keV}$ and $196 \mathrm{keV}$, respectively. This calculation is for simple weak-coupling states without the inclusion of $\Lambda-\Sigma$ coupling and calculations similar to those performed for p-shell hypernuclei remain to be performed. In addition, ${ }^{18} \mathrm{~F}$ has low-lying negative parity states that can be reached in the $\left(K^{-}, \pi^{-} \gamma\right)$ reaction [34. The lowest $0^{-}$and $1^{-}$ states are predominantly of the form $p_{1 / 2}^{-1} \times{ }^{19} \mathrm{~F}(\mathrm{gs})\left(89 \%\right.$ and $81 \%$ for $0^{-}$ and $1^{-}$in a full $1 \hbar \omega$ shell-model calculation; alternatively $72.4 \%$ and $74.6 \%$ (6 1) $\mathrm{SU}(3)$ symmetry). The $1 \hbar \omega$ hypernuclear basis requires $(s d)^{2} p_{\Lambda}$ states in addition to $p^{-1}(s d)^{3} s_{\Lambda}$ and $(s d)(p f) s_{\Lambda}$ to make a non-spurious basis. 


\section{Discussion}

Calculations using refined $(0 \hbar \omega)$ interactions for p-shell core nuclei, with the inclusion of $\Lambda-\Sigma$ coupling, have been quite successful in that a large body of data on hypernuclear level spacings has been correlated with relatively few $Y N$ parameters. The introduction of explicit $\Lambda-\Sigma$ coupling is generally beneficial. It is necessary to understand the s-shell hypernuclear binding energies, especially for ${ }_{\Lambda}^{4} \mathrm{H}$ and ${ }_{\Lambda}^{4} \mathrm{He}$. It makes significant contributions of varying size relative to the dominant $\Lambda N$ spin-spin interaction in p-shell doublet spacings and binding energies. In particular, it seems necessary to understand the groundstate doublet spacings in both ${ }_{\Lambda}^{10} \mathrm{~B}$ (a limit) and ${ }_{\Lambda}^{12} \mathrm{C}$. These effects involve the interplay of Fermi and Gamow-Teller type matrix elements connecting core states.

The remaining problems are to understand (1) the need for different $\Lambda N$ spinspin interaction strengths at either end of the p-shell and (2) the need for a stronger enhancement of the nuclear vector interaction terms (LS and ALS) near mid shell by the presence of the $\Lambda$. The next steps are to expand the shellmodel basis and to reintroduce the double one-pion exchange $\Lambda N N$ interaction considered by Gal, Soper, and Dalitz [6].

For consistency, one has to go beyond $2 \hbar \omega$ states for the core and the $\Lambda$ configurations which makes for a challenging problem. A somewhat more tractable problem is to treat the full $1 \hbar \omega$ basis of hypernuclear states [37]. This is necessary to (1) treat properly $p_{\Lambda}$ states in both the p- and sd-shells and (2) estimate decay widths for particle emission from unbound hypernuclear states [38,39, this being the way in which $\gamma$ transitions in daughter hypernuclei have been studied.

\section{Acknowledgements}

This work has been supported by the US Department of Energy under Contract No. DE-AC02-98CH10886 with Brookhaven National Laboratory.

\section{Appendix A. Structure factors for production reactions}

For a particular $l_{n} \rightarrow l_{\Lambda}$ transition, it is possible to pull out a structure factor that multiplies a particular distorted (or plane) wave radial integral and governs the relative cross sections for related states. See, for example, Section 3.2 of Ref. [40] where the structure factor is $\left(2 J_{f}+1\right) /\left(2 J_{i}+1\right)$ times the square 
of an LS one-body density-matrix (OBDME) for the transition, together with some common factors such as the square of the isospin Clebsch-Gordan coefficient for the transition. For the predominantly non-spin-flip transitions in $\left(K^{-}, \pi^{-}\right)$or $\left(\pi^{+}, K^{+}\right)$reactions, there is a single OBDME with $S=0$. In the case $\left(e, e^{\prime} K^{+}\right)$reactions, the Kroll-Ruderman term $\sigma \cdot \epsilon$ is dominant and one needs to evaluate the magnetization current contributions to the transverse electric and magnetic operators that appear in the $\left(e, e^{\prime}\right)$ cross section, specifically the $\Sigma$ and $\Sigma^{\prime}$ terms that appear in Eqs.(22b) and (22c), and given in Eqs.(1d) and (1e), of Donnelly and Haxton [41]. For the same L, we can pull out a common radial factor, basically the longitudinal form factor $F_{L}$. For the electric terms with $L=J$, we get just $F_{L}$, while for the magnetic terms we

get $\sqrt{(J+1) /(2 J+1)} F_{L}$ for $L=J-1$ and $\sqrt{J /(2 J+1)} F_{L}$ for $L=J+1$. To get the structure factors, we multiply by the OBDME with the given (LSJ), square, and add the statistical $\left(2 J_{f}+1\right) /\left(2 J_{i}+1\right)$ factor. Note that in the case of a simple particle-hole excitation for a closed-shell target nucleus, the jj OBDME is just a phase factor so that the (LSJ) OBDME is given by a normalized 9J symbol for the $j j \rightarrow L S$ transformation.

In this paper, we have just the simple $p_{N} \rightarrow s_{\Lambda}$ transition so that for $S=$ 0 we need the OBDME $(101)^{2}$, while for $S=1$ we need the combination $(111)^{2}+3 / 5(112)^{2}$. These are multiplied by $2 C^{2}\left(2 J_{f}+1\right) /\left(2 J_{i}+1\right)$, where $C$ is the isospin Clebsch-Gordan coefficient.

\section{References}

[1] D.J. Millener, Nucl. Phys. A 804 (2008) 84.

[2] H. Tamura, Nucl. Phys. A 804 (2008) 73.

[3] H. Tamura, Nucl. Phys. A 835 (2010) 3.

[4] Y. Ma et al., Nucl. Phys. A 835 (2010) 422.

[5] D.J. Millener, Nucl. Phys. A 835 (2010) 11.

[6] A. Gal, J.M. Soper, R.H. Dalitz, Ann. Phys. (N.Y.) 63 (1971) 53.

[7] A. Umeya, T. Harada, Phys. Rev. C 83 (2011) 034310.

[8] S. Cohen, D. Kurath, Nucl. Phys. 73 (1965) 1.

[9] D.J. Millener, Springer Lecture Notes in Physics, 724 (2007) 31.

[10] Y. Akaishi, T. Harada, S. Shinmura, K.S. Myint, Phys. Rev. Lett. 84 (2000) 3539.

[11] Th.A. Rijken, V.J.G. Stoks, Y. Yamamoto, Phys. Rev. C 59 (1999) 21. 
[12] M. Ukai et al., Phys. Rev. C 77 (2008) 054315.

[13] M. Ukai et al., Phys. Rev. Lett. 93 (2004) 232501.

[14] M. Agnello et al., Phys. Lett. B 681 (2009) 139.

[15] A. Gal, Nucl. Phys. A 828 (2009) 72.

[16] D.H. Davis, R. Levi Setti, M. Raymund, Nucl. Phys. 41 (1963) 73; R.H. Dalitz, Nucl. Phys. 41 (1963) 78.

[17] G. Bohm et al. Nucl. Phys. B 74 (1974) 237; D. Zieminska, R.H. Dalitz, Nucl. Phys. B 74 (1974) 248.

[18] R.E. Chrien et al., Phys. Rev. C 41 (1990) 1062.

[19] M. Ukai et al., Phys. Rev. C 73 (2006) 012501(R).

[20] M. Bejidian et al., Phys. Lett. B 94 (1980) 480.

[21] D.J. Millener, A. Gal, C.B. Dover, R.H. Dalitz, Phys. Rev. C 31 (1985) 499.

[22] L.E. Marcucci, M. Pervin, S.C. Pieper, R. Schiavilla, R.B. Wiringa, Phys. Rev. C 78 (2008) 065501.

[23] R.H. Dalitz, A. Gal. Ann. Phys. (N.Y.) 116 (1978) 167.

[24] F. Cusanno et al., Nucl. Phys. A 835 (2010) 129.

[25] K. Miwa et al., Nucl. Phys. A 754 (2005) 80c.

[26] G.-B. Liu, H.T. Fortune, Phys. Rev. C 38 (1988) 1985.

[27] M. Sotona, S. Frullani, Prog. Theor. Phys. Suppl. 117 (1994) 151.

[28] O. Hashimoto, H. Tamura, Prog. Part. Nucl. Phys. 57 (2006) 564.

[29] L. Tang, private communication.

[30] T. Motoba, M. Sotona, K. Itonaga, Prog. Theor. Phys. Suppl. 117 (1994) 123.

[31] D.H. Davis, J. Pniewski, Contemp. Phys. 27 (1986) 91; D.H. Davis, Nucl. Phys. A 754 (2005) 3c.

[32] F. Cusanno et al., Phys. Rev. Lett. 103 (2009) 202501.

[33] A.Gal, D.J. Millener, Phys. Lett. B 701 (2011) 342.

[34] H. Tamura, in this issue.

[35] W. Chung, PhD thesis, Michigan State University, 1976.

[36] J.P. Elliott, J.Phys. G 25 (1999) 577.

[37] J. Žofka, L. Majling, V.N. Fetisov, R.A. Eramzhyan, Sov. J. Part. Nucl. 22 (1991) 628. 
[38] L. Majling, R.A. Eramzhyan, V.N. Fetisov, Czech. J. Phys. 42 (1992) 1197.

[39] L. Majling, R.A. Eramzhyan, V.N. Fetisov, Phys. Part. Nucl. 28 (1997) 101.

[40] E.H. Auerbach et al., Ann. Phys. (N.Y.) 148 (1983) 381.

[41] T.W. Donnelly, W.C. Haxton, At. Data Nucl. Data Tables 23 (1979) 103. 